\title{
Assistive Device for Visually Challenged People to Read Books using Lab View
}

\author{
Sakthivel Sankaran, M Pallikonda Rajasekaran, Vishnuvarthanan govindaraj, Rameeze Raja \\ Mahaif, Guhapriyan Chinnaiah, Rajeswari Kamarajan, Ajithaa Chandagurunatha
}

\begin{abstract}
Generally, visually challenged people are unable to read books as like as a normal person. They use Braille script for reading and writing which is one of the basic techniques for them to read books and to take notes. A survey has been taken in the year 2017, in which they declared that people were completely visually impaired is around 36 million and $25 \%$ of people affected with moderate visual impairment. in average. Nearly 200 million people got affected with complete visual impairment and 1.1 billion people with near vision impairment. Nearly 5 million of visually impaired people are women. Due to various problems faced by visually challenged people several hospitals were established for giving therapy for them. Around 1,54,000 hospitals are serving for the visually challenged peoples in India. According to care survey conducted by CAGR, the growth of hospitals for visually challenged will increase more than $100 \%$ i.e. $3,25,000$ by the year 2035. Usually many devices have been developed to read books for visually challenged people but, these devices need of an external support. They are ineffective in use. In order to overcome this problem, our team has developed a device for visually challenged people to read books as like as a normal person.
\end{abstract}

Keywords : Optical character recognition, Lab VIEW, Braille Script, Visually challenged.

\section{INTRODUCTION}

$\mathrm{T}_{\mathrm{p}}$ ypically, assistive device is any device that helps to support the people who have a visually impaired problem. Lot of assistive devices were implemented for visually impaired people. Now a day's VI is used by people for assistive devices such as educational devices, mobility devices, low vision devices. Naturally, these devices have helped to read the books for visually challenged people, but these devices urge of an external support. These are inadequate in preference. In order to rectify this problem, our paper has focused on the development of a device for visually challenged people to read the books as like as a normal person. Recent

Revised Manuscript Received on December 16, 2019.

* Correspondence Author

Sakthivel Sankaran*, Department of Biomedical Engineering, Kalasalingam Academy of Research and Education, Virudhunagar, Tamilnadu, India. Email: sakthivelsankaran92@gmail.com

M Pallikonda Rajasekaran Department of Electronics and Communication Engineering, Kalasalingam Academy of Research and Education, Virudhunagar, Tamilnadu, India. Email: mpraja80@gmail.com

Vishnuvarthanan govindaraj, Department of Biomedical Engineering, Kalasalingam Academy of Research and Education, Virudhunagar, Tamilnadu, India. Email: gvvarthanan@ gmail.com

Rameeze Raja Mahaif, Guhapriyan Chinnaiah, Rajeswari Kamarajan and Ajithaa Chandagurunatha are wih the Department of Biomedical Engineering, Kalasalingam Academy of Research and Education, Virudhunagar, Tamilnadu, India.

Email: rameezeraja24@gmail.com guhaprianchinnaiah@gmail.com, rajirajan10011999333@gmail.com and ajithaaguru99@gmail.com development in mobile, computer vision, vailability of USB cameras make it feasible to assist these visually challenged persons.

\section{LITERATURE SURVEY}

[1] This method consists of three stages, in the first stage image is captured by mini camera, and in second stage, text will get filtered from the image and in final stage, extracted text will get transfer into system to get an audio as output. MATLAB software was used for image processing using mathematical operations. Input should be image such as photograph and output may be related to an image.

[2] This method, an approach that has been attempted to filter and recognize text from images and convert recognized text into speech. Image is captured from mobile camera and extracts text from image. The system of text detection and pronunciation has the audible output on mobile phone.

[3] This method comprises of three stages. In first stage, a camera gets mounted in finger is used to capture the text image from the text. In second stage, the capture image is analyzed using OCR and in third stage, a predefined dataset is loaded in order to match the observed text with the captured image. Camera which captures the images in text format dents it while sending it to OCR processing. An output is produced as audio output to read the corresponding inputs.

[4] This Method includes an embedded system for capturing an image, extracts the ROI and transforms the text into speech. By using Raspberry pi and camera as a hardware gets implemented. The extracted image undergoes a set of image processing process to isolate only the region of the image that contains the text. An embedded system that captures an image, which converts text to speech output. The output is in audio and has been achieved through raspberry pi and speakers or ear phones.

[5] This method involves the steps for transforming the text to speech converter by extracting the text from the image, which make the blind person to read the text. OCR is used.This technique makes the user to take the device anywhere at any time.

\section{TEACHING STRATEGIES}

When a teacher teaches/lectures a visually impaired or blind student, it is important that all visual materials have to be clearly explained. They should always be given oral instructions for every assignment and activity.

They should replace visual cues with audio cues. They should provide incorporate tactile learning experiences. They must always have to address students by calling their name, when they call on them to answer or ask questions. 
They should give an appropriate amount of time for VI or BS to complete their work. They should treat all of their students equally. They must consider the curriculum.

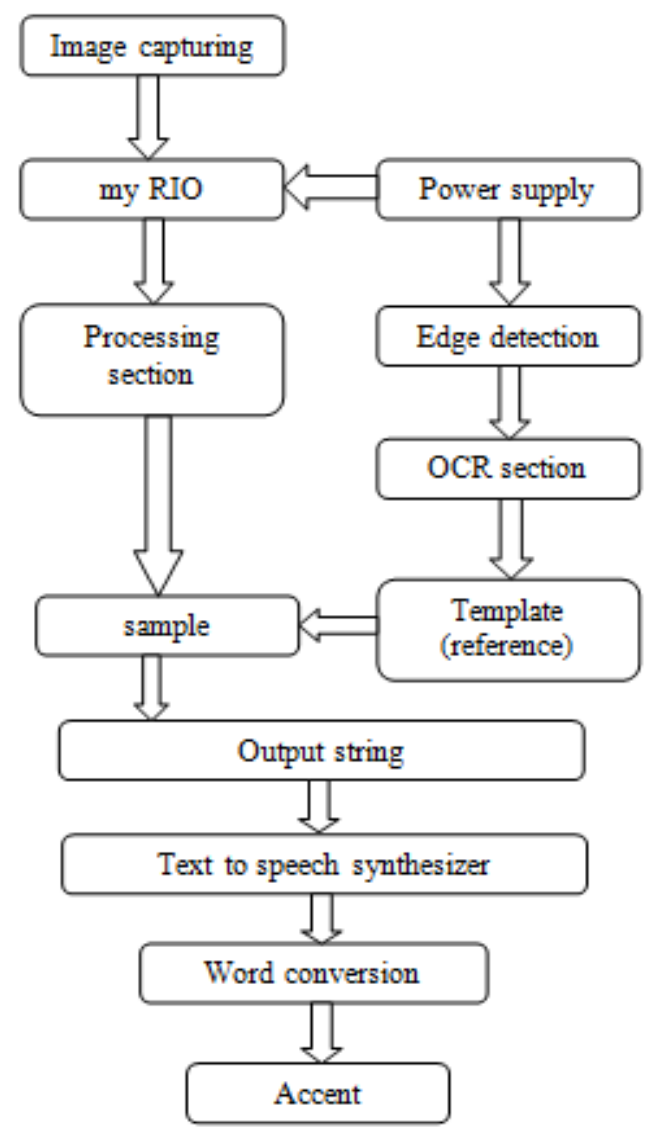

Fig. 1. Methodology of the project

\section{A. Using AIDS and Assistive Technology}

Students can record lessons using smart phones apps that can be easily downloaded for free. This one is the easiest and cheapest way to improve the learning environment for students. They provide Braille text books and handouts. These are transferred into Braille by using Braille translation software. They should provide the use of smart scanners and readers. It can be a benefit to the students who are VI and BP. These technological devices will easily convert documents into speech. The KNFB reader is an reader application, and it can be downloaded by them. It will convert printed text into speech. A page magnifier helps to enlarge the images. Text presented in any book. It can be used by students with VI. They should always use white chalk. They write with dark black marker on the white board. They avoid using colors.

\section{PROPOSED SOLUTION}

In this paper we have utilized web camera as a sensor. By using this sensor, we are going to capture the image, and it acts as the source. The camera is used as a sensor that contains text. Camera should have high resolution power. In order to get a clear image, or else the words in the image will be in the blurred format which leads to difficulty in extracting words from the image.

Lab VIEW is applied for acquisition of data process, robot control and industrial automation. Lab VIEW includes support for interfacing to devices, sensors such as camera and other devices. We can use high level, drivers that provide function nodes to LABVIEW for operating the devices.

We have made use of Lab VIEW software for acquiring the output. In Lab VIEW, we can process the actual time data without delay. We can use myRIO as an interfacing hardware. We can transfer Lab VIEW program in myRIO hardware. It is one of the advanced hardware launched by national instruments.

There is a separate area for image processing in lab VIEW. In this we use IMAQ functions in labview. It is accessible in both High level and low level VI. In this project we have used low level VI. By using OCR (optical character recognition) we are attaining or extracting the letters from the image. in this process pixels plays a major role. if there is any loss of pixel values it is difficult to extract the words. In order to overcome that we are using low level VI in which we can alter the properties so that the extraction process is a little easier and by using high resolution camera we can reduce the loss of pixels.

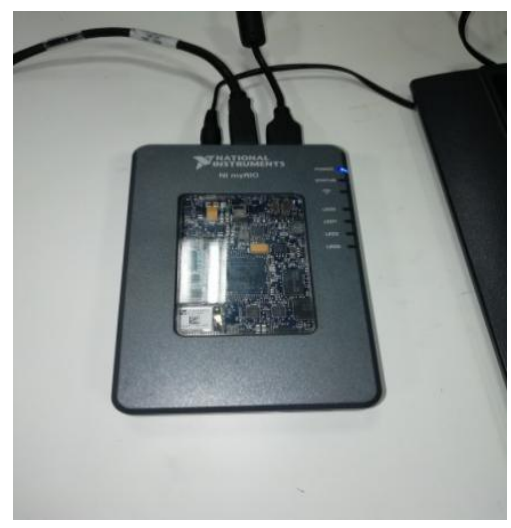

Fig. 2. myRIO an interfacing hardware

We have utilized the speakers to hear the audio output and the speakers must be compact. We can use earphones for hearing the better audio output.

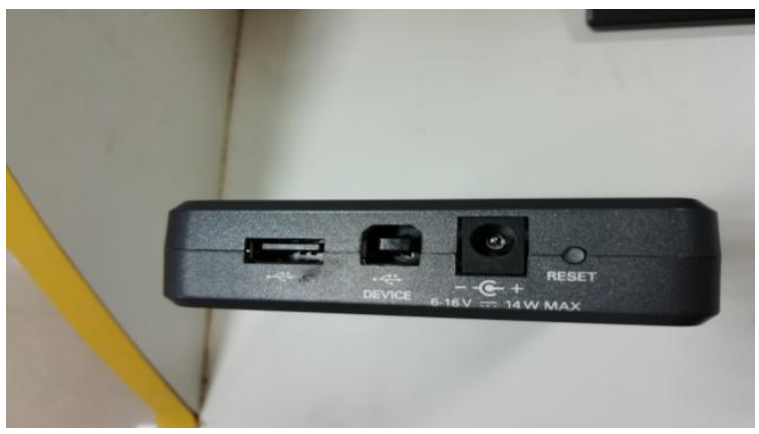

Fig. 3. Hardware port line

Camera has been used to acquire the image as an input which has been taken into the programming section by making use of a hardware. The hardware has been interfaced with a system. Camera can also connect directly to the hardware. The Lab VIEW Camera has been used to acquire the image as an input which has been taken into the programming section by making use of a program that has been developed in the system and then it has been uploaded in the hardware. In programming section, the image has been subjected into the edge 
detection. After that, we have used OCR section for extracting words from the image. We have utilized NI vision assistant for generating the template. By using template, OCR will separate and segregate the letters from images. These words will be in string format. By using text to speech synthesizer the extracted letter has been converted into a word and then accent. The output will be in audio. By using the speaker, the output can be heard clearly.

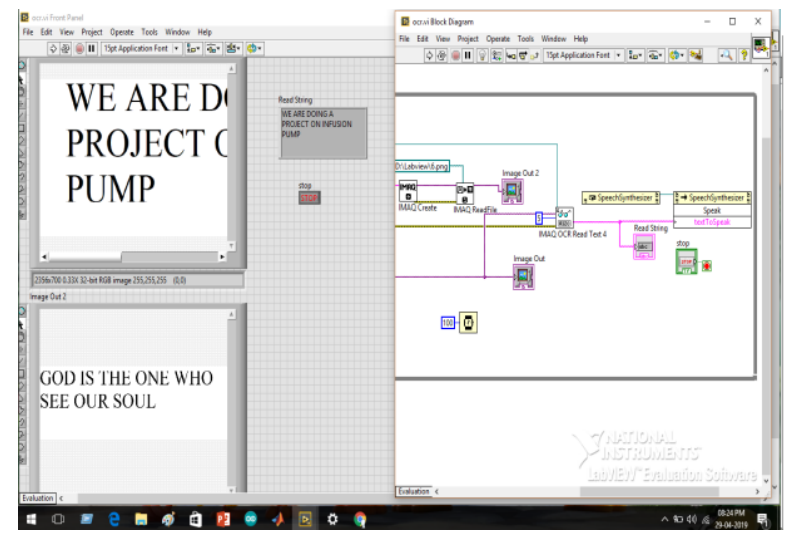

Fig. 4. Output of the Project

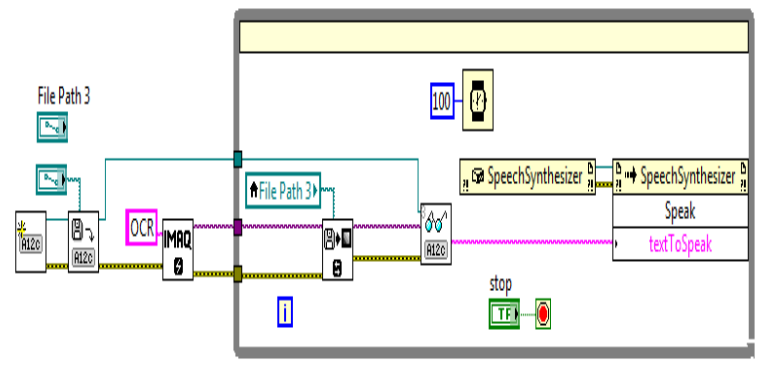

Fig. 5. Labview coding of the Project

\section{OUTPUT DESCRIPTION}

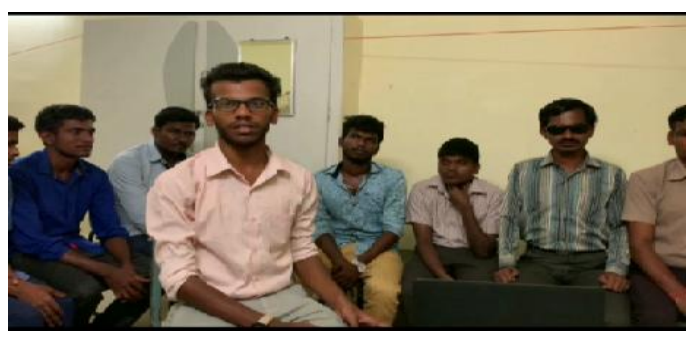

Fig.6 Interaction with visually challenged people

After stimulating the output in Lab view software we have implemented it to the community people i.e. visually challenged people. In the device, the input images have been given as jpg format, once the images has been given the program starts functioning and it convert to speech signal through headphone which can be heard by the visually challenged people.

Once hearing the audio output, visually impaired person said the words which have been listened by them are very clear and audible. By giving different input images we have checked that our system performs properly

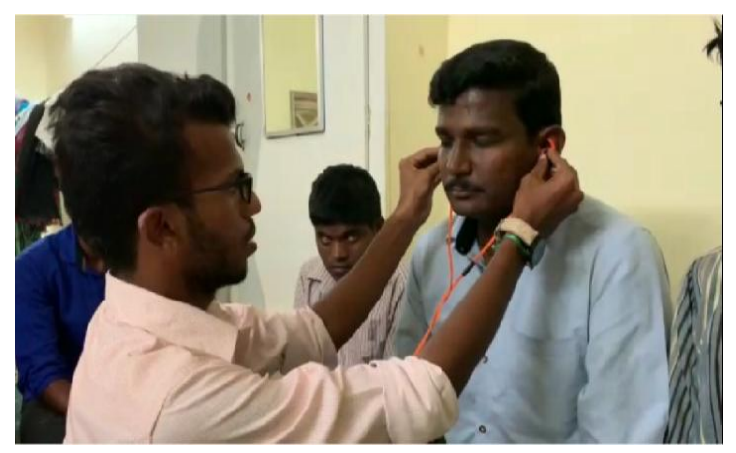

Fig.7. Simulated output to visually challenged people

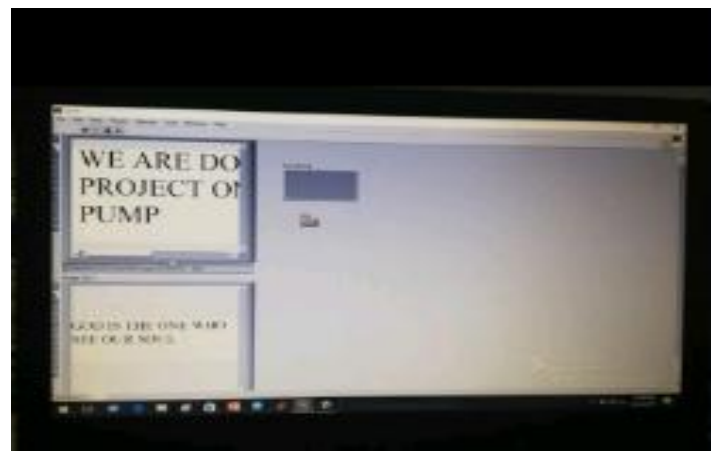

Fig. 8. Processing of input image

The input image which consists of the sentence "WE ARE DOING A PROJECT ON INFUSION PUMP" gets converted into string output which can be executed and displayed in the system. String format gets converted into audio output. The audio output can be given to the visually challenged people. The output of this work have been thoroughly verified by using different input images.

\section{CONCLUSION}

This paper presented a way to convert text signal into a speech signal. This paper gives a clear plot to design and develop a protocol that can convert the text to speech input. This prototype can convert the text to speech signal which is the output taken from the speaker. Visually impaired people use the Braille method to read the text. This method is very difficult and requires much time for practice. To overcome this, the prototype is developed in which the text is converted into a speech signal. The sound can be observed by visually impaired people. The people who are VI can understand the text in the source of sound.

\section{FUTURE WORK}

In future, we have planned to insert a hearing aid in this device which will help the visually challenged people who are deaf. This device will be very useful for them to read books. Along with this, we have planned to implement various languages, and this will be useful for them to read other language books.

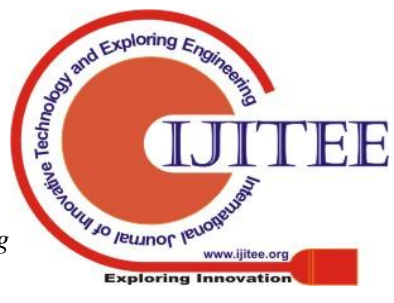




\section{REFERENCES}

1. Chucai yi and Aries Arditi "portable camera-based Assistive text and product label reading from hand-held objects for blind", vol.19,no.3, June 2014 .

2. Amarnath Singh, S.Ram kishore, G.Swathi, "portable read pad for object identification for blind through MATLAB and android" January 2015.

3. Prof.R.R Bhambare, Akashay kaul, Siddique Mohdbial, Siddarth Pandey, "Smart vision system for blind", Vol3, May 2014.

4. Dimitriob Dakopoulos and Nikolas G. Bourbakis "Wearable Obstacle Avoidance Electronic Travel Aids for Blind," Vol.40, No.1, January 2010

5. Chucai yi, Yingli tian, "Scene Text recognition in mobile application by character description and structure," IEEE Transactions on image processing, Vol 23, No.7, July 2014.

6. Nobuo Ezaki, Rarius bulau, Lambert Schomaker, "Improved text-detection methods for a camera-based text reading system for blind person," IEEE in proceedings of Eighth international and Recognition. Pp 257- 261, Vol-1, ISSN: 1520-5263,2005.

7. Marwan A.Mattar, Allen R.Hanson, Erik G. Learned-Miller, "Sign classification for the visually impaired," IEEE Workshop on Computer vision application for the visually impaired, 2005.

8. Alexander Trilla and Frances Alias.(2013), Sentence-based sentiment Analysis for Expressive Text-to speech," IEEE Transactions on Audio, Speech and Language processing,Vol-2, Issue-2,pp 223-233.

9. Balakrishnan G, Sainarayanan G, Nagarajan R and Yaacob S (2007). "Wearable real-time stereo vision for the visually impaired".Vol-14, No.2, pp 6-14.

10. Deepajose V and Sharan R (2014). "A Novel model for speech to text conversion". International referred journal of Engineering and Technology. Vol-4, No-6, pp 50-69.

11. D. Velmurugan, M.S. Sonam, S.Uma maheswari, S. Parthasarathy, K.R. Arun (2016). "A Smart reader for visually impaired people using Raspberry Pi". International journal of engineering science and computing IJE SC Volume 6. Issue No-3.

12. K. Nirmala Kumari, Meghana Reddy J (2016). "Image text to speech conversion using OCR Techinque in Raspberry pi”. International Journal of advanced Research in Electrical, electronics and instrumentation engineering. Vol-5, Issue 5,May 2016.

13. Pooja, P.Gundewar and Hemant K. Ablyankar ,"A Review on an obstacle detection in Navigation of visually impaired” International organization of scientific Research Journal of Engineering.(ISRORJEN), Vol-3. No. IPP 01-06, Jan 2013.

14. Shraga Shouel, Iwan Ulrich and johann borenstien, " Navbelt and the Guide cane", IEEE transactions on Robotics and Automation, Vol-10, No-1, pp 9-20, March 2003.

15. D.Yuvan and R.Manduchi, " Dynamic Environment Exploration using a virtual white cane". Proceedings of the IEEE computer society conference on computer vision and pattern recognition(CUPR), university of California, Santa Cruz, pp1-7, 2005.
Kalasalingam Academy of Research and Education. He had a deep involvement in Bio-signal Processing research. His work on the Image Segmentation for identification of brain tumour and image reconstruction and compression using medical images for diagnosis. Over 150 B.Tech students, 75 M.Tech students, and 8 Doctorates stand testimony for his productivity in Image Processing, Wireless Sensor Networks, and Biomedical Instrumentation research. He has so far published more than 50 papers in national and international journals and conferences. He is a Fellow of Indian Society For Technical Education (ISTE), Institute of Electrical and Electronics Engineers (IEEE), Asia-Pacific Chemical, Biological\& Environmental Engineering Society (APCBEES), Institution of Engineers (India)(IE), International Association of Engineers (IAENG) and International Association of Computer Science and Information Technology (IACSIT)

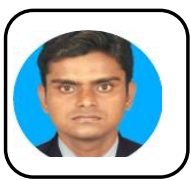

Dr.G.Vishnuvarthanan, born in 1986, has research stints in the avenues of medical image processing and artificial intelligence. He was awarded $\mathrm{PhD}$ in the year 2015 and bachelor's degree in Instrumentation and Control Engineering by 2007, and Master's Degree in VLSI by 2009. He has more than ten years of teaching and research experience and has his affiliation as Associate Professor with the Department of Biomedical Engineering of School of Bio and Chemical Sciences in the Kalasalingam Academy of Research and Education, Tamilnadu, India.

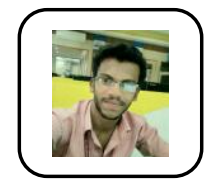

Rameeze Raja Mahaif pursuing his final year Biomedical Engineering at Kalasalingam Academy of Research and Education, Krishnankoil

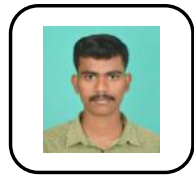

Guhapriyan Chinnaiah pursuing his final year Biomedical Engineering at Kalasalingam Academy of Research and Education, Krishnankoil.

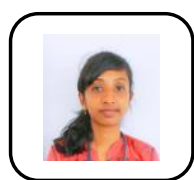

Rajeswari Kamarajan pursuing her final year Biomedical Engineering at Kalasalingam Academy of Research and Education, Krishnankoil.

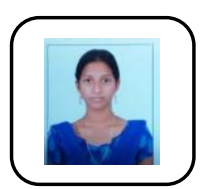

Ajithaa Chandagurunatha pursuing her final year Biomedical Engineering at Kalasalingam Academy of Research and Education, Krishnankoil.

\section{AUTHORS PROFILE}

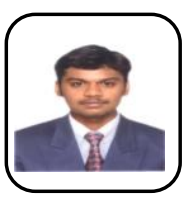

Sakthivel Sankaran received B-Eng. in Electronics and Communication Engineering, May 2013 and MEng. in (Biomedical Engineering) at VIT University, Vellore, May 2015. Currently Assistant Professor at Kalasalingam Academy of Research and Education, Krishnankoil. His research interest in Biomechanics, Rehabilitation Engineering, Biomedical Instrumentation. He has so far published more than 09 papers in national and international journals and conferences. He is a Fellow of Indian Society For Technical Education (ISTE), Institute of Electrical and Electronics Engineers (IEEE), Biomedical Engineering Society of India (BMESI) (sakthivelsankaran92@gmail.com)..

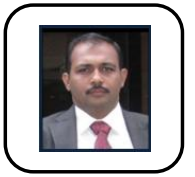

Pallikonda Rajasekaran Murugan, Born in Srivilliputhur, Virudhunagar District of Tamil Nadu in 1980, he had his schooling in the same town and graduated in Electronics and Instrumentation Engineering in 2001 from Shanmugha College of Engineering, Thanjavur and completed his M.Tech. degree in 2002 with second Rank in SASTRA University. He pursued his doctoral programme in Anna University, Chennai. Starting as a Lecturer in 2003, he became Asst. Professor in 2008, Associate Professor in 2009 and Professor in 2012 in 